\title{
Finding ordinary magic in extraordinary times: child and adolescent resilience during the COVID-19 pandemic
}

\author{
Melissa R. Dvorsky ${ }^{1}\left[\right.$ ] Rosanna Breaux ${ }^{2}$ (i) $\cdot$ Stephen P. Becker ${ }^{3}$ (i)
}

Received: 22 May 2020 / Accepted: 22 June 2020 / Published online: 1 July 2020

c) Springer-Verlag GmbH Germany, part of Springer Nature 2020

Keywords Adaptation · Youth · Coping · Coronavirus · Mental health · Resilience

The coronavirus disease 2019 (COVID-19) pandemic presents tremendous challenges to child and adolescent health. It is expected that the COVID-19 crisis, including the disease and prolonged social distancing, will have a major impact on youth well-being [1-3]. It is easy to envision how COVID-19 will be the impetus for a host of lingering negative outcomes. Schools are closed, businesses are shuttered, and families are adjusting to $24 / 7$ interaction, while caregivers simultaneously navigate parenting, financial, and professional challenges and uncertainties. As Zhou and colleagues recently reported in this journal [4], Chinese adolescents have experienced very high rates of anxiety and depression during the COVID-19 outbreak. Risk is real and warrants attention. And yet a sole focus on risk will miss resilience processes that can advance science, services, education, and policy aimed at understanding how children and adolescents respond to crisis. As such, we appreciate that Zhou and colleagues [4] also examined factors that may reduce risk for psychological distress. As Masten [5] observed two decades ago, "resilience does not come from rare or special qualities, but from the everyday magic of the ordinary, normative human resources in... children, in their families and relationships, and in their communities". This ordinary magic in children, families, and communities has a crucial role in the scientific and public health response to COVID-19.

Melissa R. Dvorsky

Melissa.Dvorsky@ucsf.edu

1 Department of Psychiatry, University of California, San Francisco, San Francisco, CA, USA

2 Department of Psychology, Virginia Polytechnic Institute and State University, Blacksburg, VA, USA

3 Division of Behavioral Medicine and Clinical Psychology, Cincinnati Children's Hospital Medical Center, Cincinnati, $\mathrm{OH}, \mathrm{USA}$
Over 50 years of research on resilience in children and adolescents implicate a range of promotive and protective factors associated with adaptation in conditions of mass adversity [6]. Resilience emerges from ordinary adaptive systems such as close relationships with competent and caring adults and peers, effective schools and communities, opportunities to succeed, and beliefs in the self. These core systems afford the capacity for self-regulation, learning, problem-solving, motivation to adapt, persistence, and hope [5]. Families, peers, schools, and communities all have a role in nurturing the growth, stability, and recovery of these systems [7]. In the midst of a global pandemic, children and adolescents depend on the resilience of these interdependent systems.

Some exposure and experience with challenges or adversity are important for developing resilience processes and growing youths' capacity and skills for handling stressful experiences [6]. In response to COVID-19, individuals, families, communities, and governments have joined together to mobilize capabilities, connect in new ways, and discover new strengths. At the individual level, the crisis, home confinement, and remote learning may provide opportunities for youth who often struggle in a traditional school environment to succeed at an individualized pace of learning, facilitating higher self-efficacy, perceived competence, and persistence. Stay-at-home orders may afford youth more time to discover new passions, hobbies, or talents, such as experiences with art, music, or nature, which can provide a greater sense of control and meaning in their lives [8]. Intrinsic curiosity and an optimistic outlook may be especially important for promoting resilience during the COVID-19 crisis [1]. Indeed, Chinese adolescents reporting a more optimistic outlook for the COVID-19 crisis had lower levels of anxiety and depression than their peers [4]. Within the family context, increased proximity provides opportunities for promoting positive family relationships [5] by engaging in collaborative 
activities (e.g., going on walks, doing puzzles/games, eating meals together). Youth may even find themselves to be the household technology expert as caregivers learn to navigate working remotely! COVID-19 has also led to communities and schools supporting youth, families, and workers by delivering meals to families, sewing facemasks, and facilitating neighborhood "birthday parades" with police cars and fire trucks.

Although the COVID-19 crisis poses significant educational, social, and mental health challenges for children and adolescents, some youth will evade these challenges entirely or experience only certain ones. Just as we need to understand which youth experience distress or difficulty during this pandemic, it is equally important to understand which youth do well and why. Notably, some youth with pre-existing difficulties will likely adapt positively [6]. For example, youth with significant anxiety may find moderate anxiety related to COVID-19 drives protective behaviors (e.g., increased compliance with hand-washing and physical distancing guidelines) that in turn further reduce anxiety [9]. Potentially in line with this possibility, Zhou and colleagues found that knowledge about the COVID-19 crisis and associated prevention/control measures may be important for mitigating psychological risk, including both anxiety and depressive symptoms [4]. Youth who have received cognitive/behavioral treatment may also be better prepared for dealing with COVID-19-related anxieties through learned strategies (e.g., deep breathing; challenging catastrophic thinking). Some youth who experience peer victimization likely find time away from school allows for greater concentration on academics, in addition to improved one-on-one interactions with peers and family, and unexpected social connections (e.g., with neighbors or distant relatives). Youth with attention and behavior challenges may find that flexibility in remote learning provides dynamic opportunities to engage with curriculum by personalizing tasks to their interests or learning style and engaging in new activities that help focus attention (e.g., breaking up tasks, listening to music/white noise) that are less feasible in typical school environments. Recognizing this heterogeneity has fueled interest in identifying potential promotive and protective mechanisms that contribute to resilient outcomes, especially among young with mental health concerns $[5,10]$. It is crucial to examine why some youth do surprisingly well, why some youth fare well in some domains but not others, and what facilitates long-term adaptation.

Longitudinal, developmentally informed research provides unique opportunities to capture both risk and resilience mechanisms among children, adolescents, and families. This is necessary to facilitate improved understanding of specific adaptive processes in response to COVID-19, particularly as stay at home regulations relax and communities and schools begin to re-open. It is critical to examine adaptation in both the short term and the long term, attending to factors that promote well-being over time and during key periods of acute and sustained stressors. Efforts to support youths' well-being during this pandemic and future crises can be improved by identifying adaptive coping and resilience-promoting processes that can inspire novel strategies to promote child and adolescent health and well-being (e.g., school-wide wellness supports). Families, schools, and communities can generate robust capacity to overcome adversity when they coordinate efforts and collaborate across levels [3]. This task may sound ordinary, but it can also be magical.

Acknowledgements Melissa Dvorsky is supported by grants (K23MH122839 and T32MH018261) from the National Institute of Mental Health (NIMH). Stephen Becker is supported by grants from the NIMH (K23MH108603) and the Institute of Education Science (IES; R305A160064, R305A160126, R305A200028). The content is solely the responsibility of the authors and does not necessarily represent the official views of the National Institutes of Health or the U.S. Department of Education

\section{Compliance with ethical standards}

Conflict of interest On behalf of all authors, the corresponding author states that there is no conflict of interest.

\section{References}

1. Xie X, Xue Q, Zhou Y et al (2020) Mental health status among children in home confinement during the coronavirus disease 2019 outbreak in Hubei Province, China. JAMA Pediatr. https ://doi.org/10.1001/jamapediatrics.2020.1619

2. Liu JJ, Bao Y, Huang X, Shi J, Lu L (2020) Mental health considerations for children quarantined because of COVID19. Lancet Child Adolesc Heal. https://doi.org/10.1016/s2352 -4642(20)30096-1

3. Cui Y, Li Y, Zheng Y, Chinese Society of Child, and Adolescent Psychiatry (2020) Mental health services for children in China during the COVID-19 pandemic: results of an expert-based national survey among child and adolescent psychiatric hospitals. Eur Child Adolesc Psychiatry. https://doi.org/10.1007/ s00787-020-01548-x

4. Zhou SJ, Zhang LG, Wang LL, Guo ZC, Wang JQ, Chen JC, Chen JX (2020) Prevalence and socio-demographic correlates of psychological health problems in Chinese adolescents during the outbreak of COVID-19. Eur Child Adolesc Psychiatry. https ://doi.org/10.1007/s00787-020-01541-4

5. Masten AS (2001) Ordinary magic resilience processes in development. Am Psychol 56(3):227-238

6. Masten AS (2015) Ordinary magic: resilience in development. Guilford Publications

7. Ungar M, Theron L (2020) Resilience and mental health: how multisystemic processes contribute to positive outcomes. Lancet Psychiatry 7(5):441-448

8. Stuckey HL, Nobel J (2010) The connection between art, healing, and public health: a review of current literature. Am J Public Health 100(2):254-263 
9. Bults M, Beaujean DJ, de Zwart O, Kok G, van Empelen P, van Steenbergen JE, Richardus JH, Voeten HA (2011) Perceived risk, anxiety, and behavioural responses of the general public during the early phase of the Influenza A (H1N1) pandemic in the Netherlands: results of three consecutive online surveys. BMC Public Health 11(1):2
10. Dvorsky MR, Langberg JM (2016) A review of factors that promote resilience in youth with ADHD and ADHD symptoms. Clin Child Fam Psychol Rev 19(4):368-391 